ORIGINAL ARTICLE

\title{
Sputum cytology of patients with severe acute respiratory syndrome (SARS)
}

\author{
G M K Tse, P-K Hui, T K F Ma, A W I Lo, K-F To, W Y Chan, L T C Chow, H-K Ng
}

J Clin Pathol 2004;57:256-259. doi: 10.1136/jcp.2003.012948

See end of article for authors' affiliations ....................

Correspondence to: Dr G M K Tse, Department of Anatomical and Cellular Pathology, Prince of Wales Hospital, Ngan Shing Street, NT, Hong Kong; garytse@cuhk.edu.hk

Accepted for publication 7 September 2003

\begin{abstract}
Background: Severe acute respiratory syndrome (SARS) is a newly described form of atypical pneumonia linked to a novel coronavirus.

Aims: To review the sputum cytology of 15 patients who fulfilled the World Health Organisation clinical criteria for SARS in an attempt to evaluate whether early diagnosis is feasible with routine sputum examination.

Methods: All sputum samples from patients with SARS from the four major hospitals in Hong Kong were reviewed; abnormalities were sought in the cellular component, including abnormal numbers and morphology of the component cells compared with those from age matched controls taken over the same period one year ago.

Results: Fifteen sputum samples from patients were compared with 25 control samples. In the patients with SARS, loose aggregates of macrophages were seen more frequently in the sputum. These macrophages frequently showed morphological changes, such as cytoplasmic foaminess, vacuole formation, and nuclear changes (including multinucleation and a ground glass appearance) when compared with the control samples.

Conclusions: The cytological features of SARS are non-specific, but the observation of any of the described features should prompt further investigations, especially in patients with suspicious clinical features.
\end{abstract}

S evere acute respiratory syndrome (SARS) is a recently described form of atypical pneumonia linked to a novel coronavirus (SARS-CoV ). ${ }^{1-4}$ The outbreak was most severe in Hong Kong and other parts of China, including Beijing and Guangzhou. During the outbreak in Hong Kong, more than 1500 patients were affected and about 200 patients died. Since July 2003, no new cases have been reported, and the World Health Organisation (WHO) has declared that SARS is now under control. Because the current understanding of SARS is still limited, it is uncertain whether resurgence of SARS will occur during the winter months. We reviewed the sputum cytology of patients diagnosed with SARS to evaluate whether diagnosis is feasible with routine sputum examination.

\section{MATERIALS AND METHODS}

Patients with a diagnosis of SARS from March to May 2003 were identified from four major hospitals in Hong Kong. The diagnostic criteria for SARS were based on the WHO recommendation. ${ }^{5}$ These clinical criteria include high fever $\left(>38^{\circ} \mathrm{C}\right)$ and respiratory symptoms, together with either a history of close contact, living within epidemic areas, or travelling in those areas within 10 days of the symptoms beginning. Investigations including the isolation of SARS$\mathrm{CoV}$ from nasopharyngeal aspirates or serological changes of increased antibody titres against SARS-CoV have also been documented. All sputum samples submitted for cytological examination from these patients were retrieved and reviewed. In patients with multiple sputum samples taken during this period, only the most cellular sample was used. The control group comprised sputum samples from age matched patients, which were taken one year ago, where there was a diagnosis of no significant pathology. Two smears were made from each sample using a standard routine laboratory procedure; these were fixed in 95\% ethanol overnight and stained with the Pap stain.
At the cytological review, the parameters assessed included the adequacy of the sample, overall cellularity, the presence of loose aggregates of more than 30 pulmonary macrophages, and any abnormal morphological changes in the macrophages. Loose aggregates of macrophages were assessed in a qualitative manner, as either present or absent. Atypical features of the macrophages included cytoplasmic foaminess, cytoplasmic vacuoles, multinucleation, and a nuclear ground glass appearance; these features were assessed semiquantitatively as the percentage of macrophages showing such changes, rounded to the nearest $5 \%$.

\section{RESULTS}

In total, 28 sputum samples from 16 patients were available, with eight patients having one sample, six patients having two samples, and two patients having four samples. Of these, one slide from each patient was selected for our study. One sputum sample was of insufficient quantity, showing only scanty squamous cells without pulmonary macrophages, and was excluded from the final analysis.

The age range of these 15 patients was 37 to 71 years (mean, 55). There were seven male and eight female patients. In nine patients, the samples were obtained within the first 10 days since the onset of symptoms, before the clinical diagnosis of SARS was made; in three patients, the samples were obtained after the diagnosis; in one patient, the sample was obtained on the day that the diagnosis was made; and in two patients, the time sequence was not known. Of these 15 patients, eight had serological confirmation, with a rising titre of antibodies against SARS-CoV, or positive viral isolation from the nasopharyngeal aspirate. In three patients, both serology and viral isolation were negative, and in the

Abbreviations: CoV, coronavirus; SARS, severe acute respiratory syndrome; WHO, World Health Organisation 
remaining four patients, only one test (either serology or viral isolation) had been done and the result was negative. Nonetheless, these last seven patients fulfilled the WHO criteria for SARS. Twenty five patients were used as controls, with one sample from each patient. The age range of the control patients was 22 to 87 years (mean, 56). There were 14 men and 11 women. In 16 of these 25 control patients, the underlying reason for sputum cytology examination was related to the lungs. Six patients had a lung shadow on $x$ ray; three patients had bronchiectasis; two patients each had haemoptysis, suspected tuberculosis, or asthma; and one patient had empyema. For the remaining patients, the presenting complaint was related to the cardiovascular system in seven patients (aneurysm, acute myocardial infarct, heart failure, and rheumatic heart disease), diabetes in one patient, and not documented in one patient.

Loose aggregates of macrophages were seen in eight of the 15 samples from patients with SARS and in three of the 25 samples from the controls (fig 1). Atypical features were seen more frequently in macrophages in the sputum from patients with SARS than in controls. Twelve of 15 sputum samples from patients with SARS and 13 from the 25 controls showed some cytoplasmic foaminess (fig 2A). In the sputum of patients with SARS, approximately $30-70 \%$ of the macrophages (mean, 50\%) showed cytoplasmic foaminess, whereas only $5-30 \%$ (mean, $13.6 \%$ ) of the macrophages showed this feature in the controls.

Well defined cytoplasmic vacuoles could be seen in some macrophages (fig 2B). Eleven of 15 sputum samples from patients with SARS and eight of 25 samples from controls showed these changes. Again, in the sputum of patients with SARS, $5-40 \%$ of the macrophages (mean, 16\%) showed cytoplasmic vacuolation, whereas only $5-10 \%$ (mean, $5.5 \%$ ) of macrophages showed this feature in the control group. In several representative cases, the slides were de-stained and re-stained with Oil Red O for lipid and periodic acid Schiff with diastase for mucin. The vacuoles were negative for both stains.

Multinucleation was found in the macrophages of 12 of 15 sputum samples from patients with SARS and six of 25 (24\%) controls (fig 2C). However, only a small proportion of the macrophages showed these changes, with a range of $5 \%$ to $15 \%$ (mean, $10 \%$ ) for patients with SARS and a range of $5 \%$ to $10 \%$ (mean, $6.4 \%$ ) for controls.

Nuclear ground glass changes were observed in the macrophages of six of the 15 sputum samples from patients

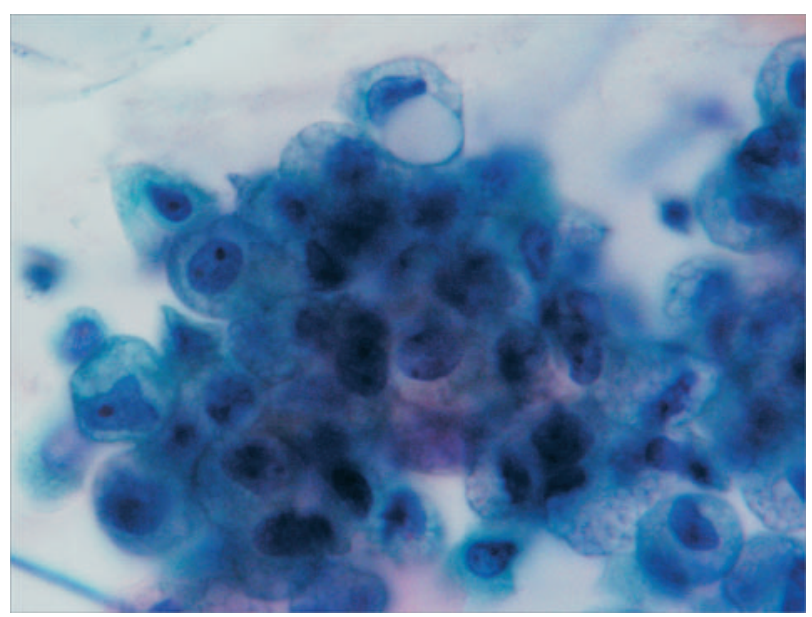

Figure 1 Aggregates of macrophages are commonly seen in sputum samples of patients with severe acute respiratory syndrome. with SARS and one of the 25 controls (fig 2D). These changes can be difficult to detect. Even in the sputum of patients with SARS, only $5-15 \%$ (mean, $7.5 \%$ ) of macrophages showed these changes, and in the single positive control case only $5 \%$ of macrophages showed such changes.

\section{DISCUSSION}

The current diagnosis of SARS is based on a set of clinical criteria, ${ }^{5}$ including fever, rapid onset of diffuse lung shadowing, plus a contact history. Laboratory confirmation has not been included in the WHO criteria for the diagnosis, as in our study. The reason for retaining just the clinical and epidemiological criteria for the case definitions is that at present there is no validated, widely and consistently available test for infection with SARS-CoV. ${ }^{5}$ Antibody tests may not become positive for three or more weeks after the onset of symptoms. Whether an antibody response is mounted in every patient is still under investigation. Molecular assays, such as reverse transcription polymerase chain reaction, must be performed using appropriate reagents and controls under strictly controlled conditions, and may not be positive in the early stages of illness using currently available reagents. Moreover, experience in other outbreak areas, such as Toronto, ${ }^{6}$ casts doubt as to the universality of laboratory evidence of SARS-CoV in the definition of this new human disease. In our series, the cytological featuressuch as cytoplasmic foaminess, vacuolation, and multinucleation of macrophages-were still valid even when we limited our analysis to the virally/serologically confirmed cases.

There is a second level of complication in case definition. The recent experience in Hong Kong showed that many patients with SARS can be afebrile or asymptomatic, and a contact history may not be easily elicited in some patients. ${ }^{7}$ SARS-CoV diagnosis is based on viral detection, isolation, or serological changes. Besides being expensive and available only in specialised hospitals, the time delay in all these methods poses a great problem in the clinical management of the patients, particularly infection containment and control. Thus, there is a role for methods that are inexpensive, quick, and easy to perform, particularly in densely populated developing countries and areas. Sputum examination is particularly suited for this purpose. In addition, sputum cytology is routinely performed for nearly all patients with chest symptoms, and an awareness of the cytological features suggestive of SARS is of the utmost importance for case recognition.

We were only able to recruit 15 cases for our study, even though four major hospitals were encompassed. These four

\section{Take home messages}

- Patients with severe acute respiratory syndrome had increased numbers of macrophages in the sputum, with the occasional formation of macrophage aggregates

- The macrophages showed morphological changes, including cytoplasmic foaminess, vacuole formation, and nuclear changes (including a ground glass appearance or multinucleation)

- These changes appear to be non-specific, and may represent either increased activation or degeneration

- Further investigations, including serological tests, are warranted when such features are seen in sputum samples 

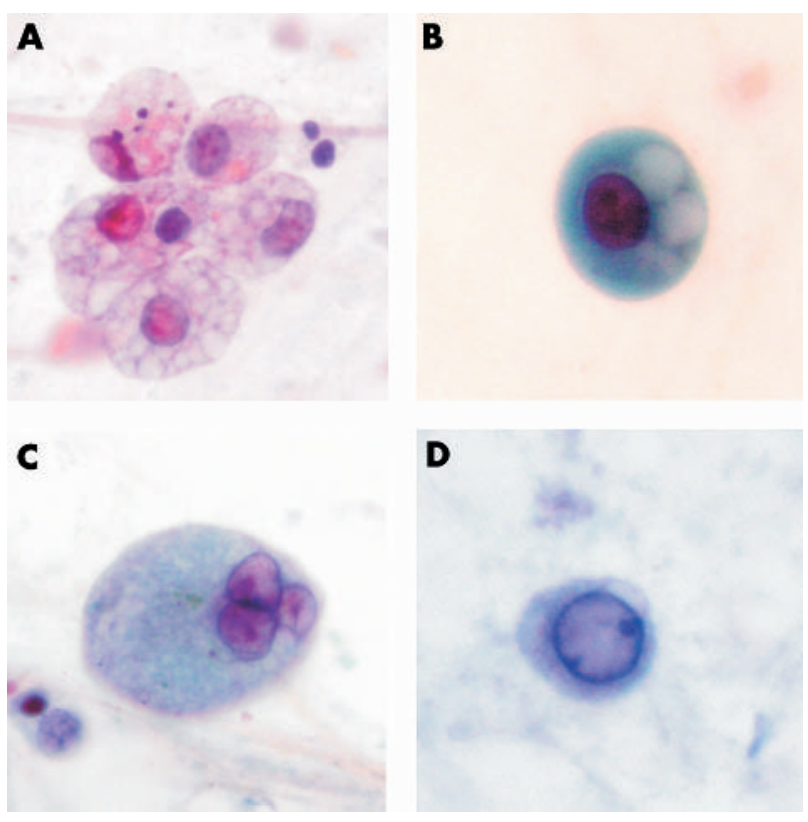

Figure 2 Morphological changes of macrophages, including (A) cytoplasmic foaminess, (B) distinct vacuoles, (C) multinucleation, and (D) ground glass appearance of the nucleus.

hospitals, with more than 480 patients with SARS, were heavily involved in the local outbreaks. The lack of available sputum samples is related to the fact that once the diagnosis was suspected, sputum samples were not collected to minimise infection risk.

\section{"Sputum cytology is routinely performed for nearly all patients with chest symptoms, and an awareness of the cytological features suggestive of severe acute respiratory syndrome is of the utmost importance for case recogni- tion"}

Despite the plethora of publications on this subject, the pathological changes of SARS in the lungs have been rarely described. ${ }^{4-10}$ In those cases uncomplicated by superimposed secondary infections, the pulmonary changes were described as predominantly those of diffuse alveolar damage, with hyaline membrane formation. There could be granulation tissue plug formation, similar to bronchiolitis obliterans organising pneumonia. Atypical pneumocytes were detected within the alveolar spaces, with enlarged nuclei and sometimes multinucleated forms, together with basophilic cytoplasm. ${ }^{4-12}$ Viral particles were demonstrated within these cells by electron microscopy. ${ }^{12}$ Increased numbers of alveolar macrophages were also described as a special feature of SARS. ${ }^{8}$

The cytological findings in the sputum were a logical extension of the pulmonary pathology. With the increased accumulation of macrophages within the alveolar spaces, aggregates of pulmonary macrophages were seen in a high proportion of sputum samples from patients with SARS. Many macrophages demonstrated foaminess in the cytoplasm, in addition to cytoplasmic vacuoles. The underlying pathogenic mechanism for this remains elusive. However, because macrophages with similar changes were also seen in the control samples (although there were fewer of them), this phenomenon is probably non-specific, and may reflect a general change in or activation of the macrophages. One of the possibilities is that cytoplasmic foaminess and/or vacuole formation may reflect changes in metabolic activation or early degeneration of the macrophages. The presence of multinucleated macrophages may be related to macrophage activation. The individual nuclei that are present within the multinucleated cells are similar to those seen in activated macrophages. The distinctive changes associated with direct viral effects (cytopathic effects)—including nuclear enlargement, basophilic nuclear staining, or formation of nuclear inclusions-were not seen. The presence of ground glass changes was unusual, and the exact nature of these changes remains to be determined.

Although the effects of SARS-CoV on macrophages are not known, other strains of human coronavirus, including 229E, and mouse coronavirus, MHV-3, have been reported to cause apoptosis in infected macrophages. Some infected macrophages do show multinucleation. ${ }^{13}{ }^{14}$ There appears to be some similarity between these effects and those observed in SARS.

Because our study was limited by the fact that we were unable to obtain sputum samples from patients with other forms of pneumonia or acute lung injury as controls, it is possible that the cytological features of these diseases overlap with those of SARS. In the literature, there have been few descriptions of the sputum cytology of acute lung injury, and this, together with the good correlation between the observed SARS cytological features and the pulmonary findings at necropsy, renders these observations useful for raising a suspicion of SARS and triggering further investigations.

In summary, we have analysed the characteristic cytological features of sputum samples from a cohort of patients with SARS. There were increased numbers of macrophages in the sputum, with the occasional formation of macrophage aggregates. The macrophages showed morphological changes, including cytoplasmic foaminess, vacuole formation, and nuclear changes. The nuclear changes included a ground glass appearance or multinucleation. The exact pathogenetic mechanism remains elusive. However, these changes appear to be non-specific, and may represent either increased activation or degeneration. These changes do not fit into any known patterns of direct viral cytopathic effects. Because these changes are non-specific, further investigations-including serological tests-are warranted when such features are observed in sputum samples.

\section{Authors' affiliations}

G M K Tse, A W I Lo, K-F To, W Y Chan, L T C Chow, H-K Ng,

Department of Anatomical and Cellular Pathology, Prince of Wales Hospital, Chinese University of Hong Kong

P-K Hui, Department of Pathology, Kwong Wah Hospital, Hong Kong T K F Ma, Department of Pathology, Alice Ho Miu Ling Nethersole Hospital, Tai Po, Hong Kong

\section{REFERENCES}

1 Peiris JS, Lai ST, Poon LL, et al. Coronavirus as a possible cause of severe acute respiratory syndrome. Lancet 2003:361:1319-25.

2 Drosten C, Gunther S, Preiser W, et al. Identification of a novel coronavirus in patients with severe acute respiratory syndrome. N Engl J Med 2003;348:1967-76.

3 Ksiazek TG, Erdman D, Goldsmith CS, et al. A novel coronavirus associated with severe acute respiratory syndrome. N Engl J Med 2003;348: 1953-66.

4 Lee N, Hui D, Wu A, et al. A major outbreak of severe acute respiratory syndrome in Hong Kong. N Engl J Med 2003;348:1986-94.

5 WHO. Case definitions for surveillance of severe acute respiratory syndrome (SARS). http://www.who.int/csr/sars/casedefinition/en/ (May 1, 2003).

6 Poutanen SM, Low DE, Henry B, et al. Identification of severe acute respiratory syndrome in Canada. N Engl J Med 2003;348:1995-2005.

7 Wong KC, Leung KS, Hui M. Severe acute respiratory syndrome (SARS) in a geriatric patient with a hip fracture. A case report. J Bone Joint Surg Am 2003;85-A: 1339-42.

8 Nicholls JM, Poon LL, Lee KC, et al. Lung pathology of fatal severe acute respiratory syndrome. Lancet 2003;361:1773-8. 
9 Franks TJ, Chong PY, Chui P, et al. Lung pathology of severe acute respiratory syndrome (SARS): a study of 8 autopsy cases from Singapore. Hum Pathol 2003;34:729

10 Ding $\mathrm{Y}, \mathrm{Wang} \mathrm{H}$, Shen $\mathrm{H}$, et al. The clinical pathology of severe acute respiratory syndrome (SARS): a report from China. J Pathol 2003:200:282-9.

11 Chan WY, Zheng J, Wang SL, et al. The pathologic changes of SARS. Zhonghua Bing Li Xue Za Zhi 2003;32:279-81.
12 Tse GM, To K-F, Chan PKS, et al. Pulmonary pathological features in cornavirus associated severe acute respiratory syndrome (SARS). J Clin Pathol 2004;57:260-5.

13 Collins AR. In vitro detection of apoptosis in monocytes/macrophages infected with human coronavirus. Clin Diagn Lab Immunol 2002;9:1392-5.

14 Belyarsky M, Belyavskaya E, Levy GA, et al. Coronavirus MHV-3-induced apoptosis in macrophages. Virology 1998;250:41-9.

\section{Clinical Evidence-Call for contributors}

Clinical Evidence is a regularly updated evidence based journal available worldwide both as a paper version and on the internet. Clinical Evidence needs to recruit a number of new contributors. Contributors are health care professionals or epidemiologists with experience in evidence based medicine and the ability to write in a concise and structured way.

\section{Currently, we are interested in finding contributors with an interest in} the following clinical areas:

Altitude sickness; Autism; Basal cell carcinoma; Breast feeding; Carbon monoxide poisoning; Cervical cancer; Cystic fibrosis; Ectopic pregnancy; Grief/bereavement; Halitosis; Hodgkins disease; Infectious mononucleosis (glandular fever); Kidney stones; Malignant melanoma (metastatic); Mesothelioma; Myeloma; Ovarian cyst; Pancreatitis (acute); Pancreatitis (chronic); Polymyalgia rheumatica; Post-partum haemorrhage; Pulmonary embolism; Recurrent miscarriage; Repetitive strain injury; Scoliosis; Seasonal affective disorder; Squint; Systemic lupus erythematosus; Testicular cancer; Varicocele; Viral meningitis; Vitiligo However, we are always looking for others, so do not let this list discourage you.

Being a contributor involves:

- Appraising the results of literature searches (performed by our Information Specialists) to identify high quality evidence for inclusion in the journal.

- Writing to a highly structured template (about 2000-3000 words), using evidence from selected studies, within 6-8 weeks of receiving the literature search results.

- Working with Clinical Evidence Editors to ensure that the text meets rigorous epidemiological and style standards.

- Updating the text every eight months to incorporate new evidence.

- Expanding the topic to include new questions once every 12-18 months.

If you would like to become a contributor for Clinical Evidence or require more information about what this involves please send your contact details and a copy of your CV, clearly stating the clinical area you are interested in, to Claire Folkes (cfolkes@bmigroup.com).

\section{Call for peer reviewers}

Clinical Evidence also needs to recruit a number of new peer reviewers specifically with an interest in the clinical areas stated above, and also others related to general practice. Peer reviewers are health care professionals or epidemiologists with experience in evidence based medicine. As a peer reviewer you would be asked for your views on the clinical relevance, validity, and accessibility of specific topics within the journal, and their usefulness to the intended audience (international generalists and health care professionals, possibly with limited statistical knowledge). Topics are usually 2000-3000 words in length and we would ask you to review between 2-5 topics per year. The peer review process takes place throughout the year, and our turnaround time for each review is ideally 10-14 days.

If you are interested in becoming a peer reviewer for Clinical Evidence, please complete the peer review questionnaire at www.clinicalevidence.com or contact Claire Folkes(cfolkes@bmigroup.com). 